\title{
TELESSAÚDE COMO FERRAMENTA EDUCACIONAL NO PROGRAMA DE RESIDÊNCIA MULTIPROFISSIONAL EM SAÚDE: RELATO DE EXPERIÊNCIA
}

\author{
TELESSAÚDE AS AN EDUCATIONAL TOOL IN THE MULTIPROFESSIONAL \\ RESIDENCE PROGRAM IN HEALTH: EXPERIENCE REPORT
} Kezia Cristina Batista dos Santos, Tamires Barradas Cavalcante, Francisca Thamires Lima de
Sousa

Universidade Federal do Maranhão - UFMA

\begin{abstract}
This study aims to report the experience of using Telehealth as an educational tool in the Multiprofessional Health Residency (RMS) program. This is an experience report about the practical aspects experienced by health professionals residing in the realization of the online EaD discipline offered by RMS and participation in tele-education activities developed by the Nucleus of Telehealth (NTS) of a university hospital in the northeast of Brazil. Brazil. The activities were carried out between February and March 2017. It was verified the effective participation of the resident professionals in all the activities proposed by the discipline, being considered differentiated and innovative. It is important to use this type of methodology to build a dynamic and collaborative teaching-learning process, providing students with autonomy, as well as coresponsibility for their learning.
\end{abstract}

Key words: Technology; Health Education; Education Distance.

\section{Resumo}

Este estudo tem como objetivo relatar a experiência da utilização do Telessaúde como ferramenta educacional no programa de Residência Multiprofissional em Saúde (RMS). Trata-se de um relato de experiência sobre os aspectos práticos vivenciados por profissionais de saúde residentes na realização de disciplina EaD online ofertada pela RMS e participação em atividades de tele-educação desenvolvidas pelo Núcleo do Telessaúde (NTS) de um hospital universitário do nordeste do Brasil. As atividades foram realizadas entre os meses de fevereiro $e$ março de 2017. Constatou-se a participação efetiva dos profissionais residentes em todas as atividades propostas pela disciplina, sendo considerada diferenciada e inovadora. Destaca-se a importância do uso deste tipo de metodologia para a construção de um processo de ensinoaprendizagem dinâmico e colaborativo, proporcionando autonomia aos discentes, assim como corresponsabilização por seu aprendizado.

Palavras chave: Tecnologia; Educação em Saúde; Educação a Distância. 


\section{Introdução}

Nas últimas décadas, as tecnologias da informação e comunicação (TIC) virtuais surgem como ferramentas importantes e como alternativas para a educação em saúde. Atualmente, as transformações nas formas de comunicação e de intercâmbio de conhecimentos, desencadeadas pelo uso generalizado das tecnologias digitais, demandam uma reformulação do processo de ensinoaprendizagem. Nesse contexto, a educação a distância (EaD) configura-se por meio do oferecimento de cursos de extensão, aperfeiçoamento, especialização e educação permanente ${ }^{1-2}$.

O Telessaúde Brasil Redes consiste na utilização de TIC para prestar serviços de saúde a distância e para compartilhar informações e conhecimento, possibilitando a interação entre profissionais de saúde ou entre estes e os usuários, bem como o acesso remoto a recursos de apoio diagnóstico ou até mesmo terapêutico ${ }^{3-}$ 4.

Foi criado em 2007, por meio da Portaria do Ministério da Saúde (MS) $n^{\circ} 35$ que lançou o Projeto-Piloto do Telessaúde Brasil, e posteriormente, redefinido e ampliado por meio da Portaria do MS $n^{\circ} 2.546$, publicada no dia 27 de outubro 2011. O Programa Nacional Telessaúde Brasil Redes possibilita o fortalecimento e a melhoria da qualidade do atendimento da atenção básica no Sistema Único de Saúde (SUS), integrando Educação Permanente em Saúde (EPS) e apoio assistencial por meio de ferramentas e tecnologias da informação e comunicação ${ }^{5-6}$.

Acrescenta-se que o Projeto Telessaúde Brasil Redes por se caracterizar um instrumento para o desenvolvimento de educação a distância, viabiliza atividades que auxiliam a qualificação de profissionais de saúde e estudantes, configurando-se como uma das estratégias da Política Nacional de Educação Permanente do SUS?.

Neste contexto, a Residência Multiprofissional em Saúde (RMS) destaca-se como um espaço intercessor ideal para o desenvolvimento da EPS, pois propicia o encontro dos profissionais residentes a partir da realização de seminários de núcleo e campo, preceptorias, aulas teóricas, atividades práticas, construção de relações e interações entre eles. Deste modo, as ações de EPS podem ser discutidas pelo grupo e incorporadas ao cotidiano de atuação de cada profissional, tornando-se presente em cada planejamento de atividade, assim como nos encontros de profissionais da saúde com docentes, usuários e profissionais do serviço ${ }^{8}$.

A RMS tem como foco a formação de profissionais de saúde críticos e reflexivos, preparados para atuar de forma integral e interdisciplinar em seus campos de atuação. Dito isto, é fundamental a introdução de modelos educativos que privilegiem as metodologias ativas, como o ensino à distância online, que rompe com o modelo tradicional, ofertando novas alternativas educacionais promovendo autonomia e autossuficiência ao aluno. Diante disto, objetivou-se, com este estudo, relatar a experiência da utilização do Telessaúde como ferramenta educacional no programa de Residência Multiprofissional em Saúde (RMS).

\section{Metodologia}

O presente trabalho consiste em um relato de experiência sobre os aspectos práticos vivenciados por profissionais de saúde residentes na realização da disciplina EaD online "Introdução à Bioestatística" ofertada pela RMS e participação em atividades de tele-educação desenvolvidas pelo Núcleo do Telessaúde (NTS) de um hospital universitário do nordeste do Brasil.

As atividades foram desenvolvidas entre fevereiro e março de 2017 com profissionais residentes das seguintes categorias: enfermagem, fisioterapia, farmácia, nutrição, fonoaudiologia, terapia ocupacional, odontologia, assistência social, psicologia e educação física ativos e matriculados na referida disciplina.

O local do estudo é gerenciado pela Empresa Brasileira de Serviços Hospitalares (EBSERH) desde 2013, sendo um hospital de perfil terciário, referência estadual para procedimentos de alta complexidade em diversas áreas e especialidades, vinculado ao Sistema Único de Saúde (SUS). Possui Núcleo de Telessaúde (NTS), criado em 2007, responsável pela promoção de atividades de Telessaúde e educação à distância, que conta com recursos humanos e tecnológicos que permitem o suporte a atividades presenciais e à distância, seja por meio de vídeo ou webconferência.

A construção desse relato parte de reflexões teóricas provenientes da vivência prática durante a realização da disciplina, onde se discutem as diferenças e similaridades entre o processo de ensino-aprendizagem tradicional e à distância EaD online, habilidades e competências adquiridas, assim como a contribuição da 
metodologia utilizada para a formação do profissional de saúde residente. Os registros documentais, tais como relatórios de atividades, documentação de configuração e registro do curso no AVA e instrumentos de avaliação e autoavaliação foram as fontes com base nas quais a experiência foi analisada e descrita.

\section{Resultados e Discussões}

Seguindo a legislação vigente, Resolução CNRMS n ㄱ, de 4 de maio de $2010^{9}$, que dispõe sobre a duração e a carga horária dos programas de Residência Multiprofissional em Saúde e sobre a avaliação e a frequência dos profissionais da saúde residentes, os Programas de Residência Multiprofissional em Saúde têm a duração mínima de dois anos, equivalente a uma carga horária mínima total de 5760 (cinco mil, setecentos e sessenta) horas. Destas, $80 \%$ devem ser direcionadas para o desenvolvimento de atividades práticas e $20 \%$ para atividades teóricas ou teórico-práticas. Atividades teóricas são aquelas cuja aprendizagem se desenvolve por meio de estudos individuais e em grupo, em que o profissional de saúde residente conta, formalmente, com a orientação de docentes, preceptores ou convidados, visando à aquisição de conhecimentos teóricos e técnicos que possibilitem a elaboração de modelos teóricopráticos.

A disciplina "Introdução à Bioestatística" corresponde a uma atividade teórica da RMS, componente obrigatório curricular com carga horária de 30 horas, anteriormente ministrada de forma $100 \%$ presencial. As autoras iniciaram o contato com o Telessaúde a partir da introdução da referida disciplina sob forma $\mathrm{EaD}$ online, modalidade até então inédita quando comparada às outras disciplinas convencionais e presenciais. A disciplina foi reformulada para formato de curso autoinstrucional, contando com três encontros presenciais, o primeiro a saber, para apresentação do curso e instruções acerca da plataforma do Ambiente Virtual de Aprendizagem (AVA) com orientação referente ao primeiro acesso, cadastro, realização de atividades online, testes avaliativos, tutoria, chats, fóruns, etc; o segundo para avaliação da turma quanto ao andamento do curso; e o terceiro para encerramento da disciplina e feedback do processo de aprendizagem.

Quanto às atividades de tele-educação, foram realizados seminários virtuais (webconferências) com os professores tutores sobre temas relacionados ao conteúdo programático da disciplina, em dia e horário previamente agendados, em momento que correspondesse às atividades de dispersão dos profissionais residentes, sendo contabilizadas como atividades teóricas. Os seminários virtuais foram realizados na sala virtual do Telessaúde, disponibilizada pela Rede Universitária de Telemedicina (RUTE) e transmitido de forma online.

Atualmente, entende-se que os procedimentos de ensino são tão importantes quanto os próprios conteúdos de aprendizagem $^{10}$. Ao longo dos últimos anos, as técnicas tradicionais de ensino vêm mostrando várias fragilidades apresentando necessidades de reformulação ${ }^{11}$. Neste sentido, diversos estudos apontam que o uso de novas tecnologias de informação e comunicação favorecem a construção de um processo de ensinoaprendizagem ativo e significativo ${ }^{1,11-12}$.

$A$ educação à distância (EaD) é a modalidade educacional na qual a mediação didático-pedagógica nos processos de ensino e aprendizagem ocorre com a utilização de meios e tecnologias de informação e comunicação, com estudantes e professores desenvolvendo atividades educativas em lugares ou tempos diversos. Essa modalidade é regulada por uma legislação específica e pode ser implantada na educação básica e na educação superior ${ }^{13}$.

O ensino EaD propicia a possibilidade de autoformação e a disseminação de conhecimentos em larga escala, além de favorecer a comunicação, a interação entre pares e a troca de experiências práticas ${ }^{14}$. Tal modalidade de ensino ganhou uma nova perspectiva com o aparecimento das Tecnologias de Informação e Comunicação (TICS), facilitando o acesso ao ensino devido à sofisticação, rapidez e melhor aproximação entre professor/tutor e aluno $^{12}$. Fato este, se traduz de forma positiva no cotidiano do profissional residente que exerce rotineiramente carga horária de 60 horas semanais no treinamento em serviço (TS), proporcionando, maior flexibilidade de tempo para atenção e dedicação aos estudos e atividades teóricas.

Observam-se na literatura diversos mecanismos de estruturação de processos formativos EaD com utilização das TIC, um dos mais utilizados refere-se ao ambiente virtual de aprendizagem (AVA) ou Learning Management Systems (LMS sistemas de gerenciamento de aprendizagem), ambiente que viabiliza a interação autônoma do aluno mediante o desenvolvimento de atividades pedagógicas, permitindo-o construir seu conhecimento. Estes ambientes disponibilizam ao usuário, professor e 
aluno, vários recursos que podem ser utilizados como estratégias de ensino. Fazem parte destes recursos o chat, o fórum, e-mail, listas de discussão, blogs e tele e webconferência, etc ${ }^{1}$.

É importante frisarmos o alto índice de profissionais residentes que acessaram as webconferências por meio do ambiente virtual de aprendizagem. Tal índice pode ser justificado pela inovação no método de ensino adotado e pela possibilidade de participação ativa em momentos de discussão fora do treinamento em serviço, onde se era possível expressar ideias, opiniões e expor dúvidas.

O potencial pedagógico da webconferência surge como ferramenta que proporciona um elo construtivo e dinâmico entre professor e aluno. Esta metodologia tem evoluído e trazido um conjunto de funcionalidades que favorecem seu uso em ambientes acadêmicos, devido à considerável economia de tempo e recursos para reunir professores e estudantes e desenvolver atividades em equipes. Possui inúmeras ferramentas de comunicação em um mesmo ambiente, permitindo interações multidirecionais por voz, texto (chat) e vídeo, além do compartilhamento de arquivos, oferecendo interação instantânea e intensificação da presença virtual do professor-tutor a distância ${ }^{14}$. É importante ressaltar que o aluno precisará desenvolver fluência tecnológica e, cada vez mais, sofisticada, para o uso destas tecnologias frente à realidade da globalização e dos processos formativos atuais ${ }^{15}$.

As atividades presenciais também foram de extrema importância para o aumento efetivo da participação dos profissionais residentes ao curso. No segundo encontro os professores tutores reuniram os discentes para avaliar o andamento da disciplina, orientando quanto às dúvidas e dificuldades encontradas. O terceiro encontro ocorreu após a conclusão do curso online e representou o encerramento da disciplina com avaliação e feedback do processo de ensino-aprendizagem. Algumas dificuldades foram citadas: falta da tradicional relação presencial de professor e aluno; dificuldade de lidar com as novas tecnologias; ausência de reciprocidade da comunicação, foram as mais importantes, entretanto, a experiência foi bastante exitosa, sendo a disciplina considerada diferenciada e inovadora.

\section{Conclusões}

Constatou-se a participação efetiva dos profissionais residentes em todas as atividades propostas pela disciplina ministrada por meio do
Telessaúde, sendo considerada diferenciada e inovadora. A educação à distância é uma poderosa ferramenta para a prática de saúde e colabora como uma nova estratégia de aprendizado na busca de um processo de ensinoaprendizagem mais eficiente e mais eficaz.

Destaca-se a importância do uso das tecnologias de informação e comunicação no processo de formação profissional como instrumento pedagógico, proporcionando aprendizagem dinâmica e colaborativa, assim como autonomia aos discentes e corresponsabilização por seu aprendizado.

\section{Referências}

1. Rodrigues RCV, Peres HHC. Panorama brasileiro do ensino de enfermagem a distância. Rev Esc Enferm USP. [Internet] 2008 [acesso em 12 de janeiro de 2019];42(2):298-304. Disponível em:

http://www.scielo.br/scielo.php?script=sci_artte xt\&pid=S0080-62342008000200013

2. Oliveira C, Moura SP, Sousa ER. TIC's na Educação: A utilização das tecnologias da informação e comunicação na aprendizagem do aluno. Pedagogia em Ação. [Internet] 2015 [acesso em 12 de janeiro de 2019];7(1):1-21. Disponível em: http://periodicos.pucminas.br/ index.php/pedagogiacao/article/view/11019/886 4

3. Celes RS, Rossi TRA, Barros SG, Santos CML, Cardoso C. A telessaúde como estratégia de resposta do Estado: revisão sistemática. Rev Panam Salud Publica. [Internet] 2018 [acesso em 02 de janeiro de 2019];42:e84. Disponível em: http://iris.paho.org/xmlui/bitstream/handle/ 123456789/49154/v42e842018.pdf?sequence=1 4. Correia AD, Dobashi BF, Gonçalves CC, Monreal VR, Nunes EA, Haddad PO, et al. Teleodontologia no programa nacional telessaúde Brasil redes: relato da experiência em Mato Grosso Do Sul. Rev ABENO. [Internet] 2014 [acesso em 29 dezembro de 2018];14(1):17-29. Disponível em: https://revabeno.emnuvens.com.br/revabeno/ar ticle/view/96

5. Brasil. Ministério da Saúde. Portaria no 35, de 4 de janeiro de 2007. Institui, no âmbito do Ministério da Saúde, o Programa Nacional de Telessaúde. Diário Oficial da União. Brasília, DF: Ministério da Saúde, 2007. Seção 1.

6. Brasil. Ministério da Saúde. Portaria no 2.546, de 27 de outubro de 2011. Redefine e amplia o Programa Telessaúde Brasil, que passa a ser denominado Programa Nacional Telessaúde Brasil Redes (Telessaúde Brasil Redes). Diário 
Oficial da União. Brasília, DF: Ministério da Saúde, 2011.

7. Oliveira MAN. Educação a distância como estratégia para educação permanente em saúde: possibilidades e desafios. Rev Bras Enferm. [Internet] 2007 [acesso em 10 de janeiro de 2019];60(5):585-9. Disponível em: http://www.scielo.br/scielo.php?pid=s00347167 2 007000500019\&script=sci_abstract\&tlng=pt

8. Silva $\mathrm{CT}$, Terra MG, Kruse MHL, Camponogara S, Xavier MS. Residência multiprofissional como espaço intercessor para a educação permanente em saúde. Texto Contexto Enferm. [Internet] 2016 [acesso em 11 de janeiro de 2019];25(1):e2760014. Disponível em: http://www.scielo.br/pdf/tce/v25n1/0104-0707tce-25-01-2760014.pdf

9. Brasil. Comissão Nacional de Residência Multiprofissional em Saúde. Resolução CNRMS no 3 , de 4 de maio de 2010. Dispõe sobre a duração e a carga horária dos programas de Residência Multiprofissional em Saúde e de Residência em Área Profissional da Saúde e sobre a avaliação e a frequência dos profissionais da saúde residentes. [Internet] Diário Oficial da União [acesso em 10 de janeiro de 2019]; Poder Executivo, Brasília: Distrito Federal. Seção I, p. 14-15. Disponível em: http://portal.mec.gov.br/index.php?option=com _docman\&view =download\&alias=15449-resolcnrms-n3-04maio-2010\&Itemid =30192

10. Paiva MRF, Parente JRF, Brandão IR, Queiroz AHB. Metodologias ativas de ensinoaprendizagem: revisão integrativa. SANARE. [Internet] 2016 [acesso em 12 de janeiro de 2019];15(2):145-153. Disponível em: https://sanare.emnuvens.com.br/sanare/article/ view /1049

11. Faria MGA, David HMSL. Enfermagem e educação permanente a distância: o exemplo do projeto Telessaúde Brasil, núcleo Rio de Janeiro. Cogitare Enferm. [Internet] 2010 [acesso em 08 de janeiro de 2019];15(4):667-73. Disponível em: https://revistas.ufpr.br/cogitare/article/view/203 63

12. Tanaka EZ, Sartori DVB, Ferreira LR, Bermejo L. A educação a distância nos cursos de graduação em enfermagem: aplicação e efetividade. Rev. on line de Política e Gestão Educacional. [Internet] 2017 [acesso em 11 de janeiro de 2019];21(1):831-841. Disponível em: https://periodicos.fclar.unesp.br/rpge/article/vie wFile/10455/6813

13. Brasil. Decreto 5.622, de 19 de dezembro de 2005. Regulamenta o artigo 80 da Lei no 9.394, de 20 de dezembro de 1996, que estabelece as diretrizes e bases da educação nacional. [Internet]. Diário Oficial [da] República
Federativa do Brasil [acesso em 11 de janeiro de 2019]. Brasília, DF, 20 dez. 2005. Disponível em: http://www.planalto.gov.br/ccivil_03/_Ato20042006/2005/Decreto/D5622.htm

14. Azambuja EG. O uso do vídeo e da web conferência em EAD. Revista Cesuca Virtual: Conhecimento sem Fronteiras. [Internet] 2015 [acesso em 13 de janeiro de 2019];2(3):82-92. Disponível em: ojs.cesuca.edu.br/index.php/cesucavirtual/article /view/909/674

15. Demo P. Aprender a aprender Neoliberal? Rev. Ciências Humanas. [Internet] 2013 [acesso em 13 de janeiro de 2019];14(22):25-53. Disponível em: http://revistas.fw.uri.br/index.php/revistadech/a rticle/view/983/1431

\section{Endereço para Correspondência}

Av. dos Portugueses, 1966 - Vila Bacanga, São Luís - MA

CEP.: 65080-805

e-mail: kezia_cristinabs@hotmail.com

Recebido em 30/01/2019

Aprovado em 06/02/2019

Publicado em 13/08/2019 